\title{
Anti-interference Tracking Algorithm Based on Structural Random Jump System Theory
}

\author{
Xuchao Kang ${ }^{1}$, Guangjun $\mathrm{He}^{1,{ }^{*}}$, Feng Chen ${ }^{1}$ and Qifang $\mathrm{He}^{2}$ \\ ${ }^{1}$ Air and Missile Defence College, Air Force Engineering University, Xi'an 710051, China \\ ${ }^{2}$ Information and Navigation College, Air Force Engineering University, Xi'an 710051, China \\ ${ }^{*}$ Corresponding author
}

\begin{abstract}
In the process of tracking a maneuvering target, when there is interference, the system parameters and structure will randomly jump. The previous algorithms have less consideration. The thesis applies the structural random jump system theory to the anti-jamming tracking problem of the two sensors, establishes its nonlinear model, completes the algorithm design, and completes the linearization of the nonlinear model. Experimental verification shows that it can effectively solve the problem of stable tracking of the target in the presence of interference and when the two different sensors are switched to each other.
\end{abstract}

Keywords-maneuvering target tracking; random transition system; nonlinear filtering

\section{INTRODUCTION}

In the process of maneuvering target tracking, there often occurs a situation where the system parameters and structures randomly jump, that is, there are uncertainties in parameters and structures in the system. The system with this characteristic of uncertainty is called system with random changing structure, (SRCS).

Especially in the presence of interference, the parameters and structural uncertainty of the maneuvering target tracking system increase significantly. However, in previous studies, this factor was rarely considered. Under this background, the structure random jump system theory is applied to the antijamming tracking problem of the two sensors to effectively deal with the enemy's various interference measures, achieve accurate and stable tracking of the target, and provide a new idea for the maneuvering target tracking.

\section{ANTI-JAMMING TRACKING ALGORITHM BASED ON NONLINEAR GAUSSIAN APPROXIMATION FILTER OF SRCS SYSTEM}

Due to factors such as interference and random switching of several sensors, the structure or parameters of the system may change drastically. In online Gaussian conditions, the target motion equation can be described as

$$
\boldsymbol{x}(k+1)=\boldsymbol{\Phi}(s, k) \boldsymbol{x}(k)+\boldsymbol{w}(s, k)
$$

Its observation equation can be described as

$$
\boldsymbol{z}(k+1)=\boldsymbol{H}(s, k+1) \boldsymbol{x}(k+1)+\boldsymbol{v}(s, k+1)(s \in S=\overline{1, M})
$$

In the formula: $\boldsymbol{x}(k)$ is the n-dimensional target motion state vector, $\boldsymbol{z}(k)$ is the m-dimensional observation vector, $\boldsymbol{w}(s, k)$ and $\boldsymbol{v}(s, k)$ are m-dimensional and n-dimensional white noise vectors, respectively, $s$ is the structural label of the system which is described by $m$ finite state conditional Markov chains. $\boldsymbol{\Phi}(s, k)$ and $\boldsymbol{H}(s, k)$ are $n \times n$-dimensional and $m \times n$-dimensional known function matrices

Now suppose that all noises are Gaussian random sequences that are independent of each other and have a mean value of zero. The initial values of the target motion state are also Gaussian and independent of all noises. That is to say,

$$
\left\{\begin{array}{l}
\boldsymbol{w}(s, k) \sim \boldsymbol{N}[\boldsymbol{w}(s, k) \mid \boldsymbol{\mu}(s, k), \boldsymbol{Q}(s, k)] \\
\boldsymbol{v}(s, k) \sim \boldsymbol{N}[\boldsymbol{v}(s, k) \mid \boldsymbol{\varepsilon}(s, k), \boldsymbol{R}(s, k)] \\
\boldsymbol{x}_{\boldsymbol{0}}^{(s)} \sim \boldsymbol{N}\left[\boldsymbol{x}_{\boldsymbol{0}}^{(s)} \mid \boldsymbol{m}_{0}^{(s)}, \Theta_{0}^{(s)}\right]
\end{array}\right.
$$

In the formula: $\boldsymbol{x}_{\boldsymbol{0}}^{(s)}$ is the initial state of the target under the $S$ structure, The function $N\left\{\alpha \mid \bar{\alpha}, E_{\alpha}\right\}$ indicates that the random vector has a Gaussian distribution with a mean of $\bar{\alpha}$ and a variance of $\boldsymbol{E}_{\alpha}$

let $s=s(k+1), r=s(k)$

$$
\boldsymbol{z}_{k+1}=\boldsymbol{z}(k+1) \boldsymbol{Z}^{k}=[\boldsymbol{z}(0), \boldsymbol{z}(1), \mathrm{L}, \boldsymbol{z}(k-1), \boldsymbol{z}(k)],
$$

And ignore the moments $k$ that do not cause ambiguity.

According to equation of state (1), observation equation (2) and prior condition (3), The conditional transition probability density function $f[\boldsymbol{x}(k+1) \mid \boldsymbol{x}, r]$ of the target motion state $\boldsymbol{x}(k+1)$ and The conditional probability density function $f\left[\boldsymbol{z}_{k+1} \mid \boldsymbol{x}(k+1), s\right]$ of the observation vector $\boldsymbol{z}(k+1)$ is 


$$
\begin{aligned}
& f[\boldsymbol{x}(k+1) \mid \boldsymbol{x}, r]=f[\boldsymbol{x}(k+1) \mid \boldsymbol{x}(k), s(k)] \\
& \quad=\boldsymbol{N}[\boldsymbol{x}(k+1) \mid \boldsymbol{\Phi}(r, k) \boldsymbol{x}(k \mid r, k)+\boldsymbol{\mu}(r, k), \boldsymbol{Q}(r, k)] \\
& f\left[\boldsymbol{z}_{k+1} \mid \boldsymbol{x}(k+1), s\right]= \\
& \boldsymbol{N}\left[\boldsymbol{z}_{k+1} \mid \boldsymbol{H}(s, k+1) \boldsymbol{x}(k+1 \mid s, k)+\boldsymbol{\varepsilon}(s, k+1), \boldsymbol{R}(s, k+1)\right]
\end{aligned}
$$

Assuming that the hopping process of the structure is independent of the system state and depends only on the structural state of the previous step system, the transition probability of the structural state is established as follows:

$$
q[s, k+1 \mid x(k), r, k] \equiv q[s, k+1 \mid r, k]=q^{(s r)}(k+1, k)
$$

Due to the change of the system structure, the distribution of the target motion state is no longer Gaussian. Therefore, for the conditional probability density $f\left[\boldsymbol{x}(k) \mid r, \boldsymbol{Z}^{k}\right]$, the Gaussian approximation method is used to obtain the following relationship.

$$
f\left[\boldsymbol{x}(k) \mid r, \boldsymbol{Z}^{k}\right]=N[\boldsymbol{x}(k) \mid \boldsymbol{x}(k \mid r, k), \boldsymbol{P}(k \mid r, k)]
$$

Through the discretized optimal filter equations, the tracking algorithm equations are deduced.

State prediction:

$$
\boldsymbol{x}(k+1 \mid r, k)=\boldsymbol{\Phi}(r, k) \boldsymbol{x}(k \mid r, k)+\boldsymbol{\mu}(r, k)
$$

Covariance prediction:

$$
\boldsymbol{P}(k+1 \mid r, k)=\boldsymbol{\Phi}(r, k) \boldsymbol{P}(k \mid r, k) \boldsymbol{\Phi}^{T}(r, k)+\boldsymbol{Q}(r, k)
$$

Mixed state prediction:

$$
\boldsymbol{x}(s, k+1 \mid r, k)=\boldsymbol{x}(k+1 \mid r, k)+\boldsymbol{K}_{k+1}^{(s r)} \boldsymbol{e}_{k+1}^{(s r)}
$$

Mixed covariance prediction:

$$
\boldsymbol{P}(s, k+1 \mid r, k)=\left[\boldsymbol{I}-\boldsymbol{K}_{k+1}^{(s r)} \boldsymbol{H}(s, k+1)\right] \boldsymbol{P}(k+1 \mid r, k)
$$

State estimation:

$$
\begin{aligned}
& \boldsymbol{x}(k+1 \mid s, k+1)= \\
& \frac{\sum_{r=1}^{M} q^{(s r)}(k+1, k) \boldsymbol{N}\left[\boldsymbol{e}_{k+1}^{(s r)} \mid 0, \boldsymbol{\Xi}_{e k+1}^{(s r)}\right] f\left(r \mid \boldsymbol{Z}^{k}\right) \boldsymbol{x}(s, k+1 \mid r, k)}{\sum_{r=1}^{M} q^{(s r)}(k+1, k) \boldsymbol{N}\left[\boldsymbol{e}_{k+1}^{(s r)} \mid 0, \boldsymbol{\Xi}_{e k+1}^{(s r)}\right] f\left(r \mid \boldsymbol{Z}^{k}\right)}
\end{aligned}
$$

Covariance estimation:

$$
\begin{aligned}
& \boldsymbol{P}(k+1 \mid s, k+1)= \\
& \frac{\sum_{r=1}^{M} q^{(s r)}(k+1, k) \boldsymbol{N}\left[\boldsymbol{e}_{k+1}^{(s r)} \mid 0, \boldsymbol{\Xi}_{e k+1}^{(s r)}\right] f\left(r \mid \boldsymbol{Z}^{k+1}\right)}{\sum_{r=1}^{M} q^{(s r)}(k+1, k) \boldsymbol{N}\left[\boldsymbol{e}_{k+1}^{(s r)} \mid 0, \boldsymbol{\Xi}_{e k+1}^{(s r)}\right] f\left(r \mid \boldsymbol{Z}^{k+1}\right)}\{\boldsymbol{P}(s, k+1 \mid r, k) \\
& \left.+[\boldsymbol{x}(s, k+1 \mid r, k)-\boldsymbol{x}(k+1 \mid s, k)] \cdot[\boldsymbol{x}(s, k+1 \mid r, k)-\boldsymbol{x}(k+1 \mid s, k)]^{T}\right\}
\end{aligned}
$$

State estimation synthesis:

$$
\boldsymbol{x}(k+1 \mid k+1)=\sum_{s=1}^{M} f\left(s \mid \boldsymbol{Z}^{k+1}\right) \boldsymbol{x}(k+1 \mid s, k+1)
$$

Covariance estimation synthesis:

$$
\begin{aligned}
& \boldsymbol{P}(k+1 \mid k+1)=\sum_{s=1}^{M} f\left(s \mid \boldsymbol{Z}^{k+1}\right)\{\boldsymbol{P}(k+1 \mid s, k+1) \\
& \left.+[\boldsymbol{x}(k+1 \mid s, k+1)-\boldsymbol{x}(k+1)][\boldsymbol{x}(k+1 \mid s, k+1)-\boldsymbol{x}(k+1)]^{T}\right\}
\end{aligned}
$$

Conditional probability density function of system structure state:

$$
f\left(s \mid \boldsymbol{Z}^{k+1}\right)=\frac{\sum_{r=1}^{M} q^{(s r)}(k+1, k) \boldsymbol{N}\left[\boldsymbol{e}_{k+1}^{(s r)} \mid 0, \boldsymbol{\Xi}_{e k+1}^{(s r)}\right] f\left(r \mid \boldsymbol{Z}^{k}\right)}{\sum_{s=1}^{M} \sum_{r=1}^{M} q^{(s r)}(k+1, k) \boldsymbol{N}\left[\boldsymbol{e}_{k+1}^{(s r)} \mid 0, \boldsymbol{\Xi}_{e k+1}^{(s r)}\right] f\left(r \mid \boldsymbol{Z}^{k}\right)}
$$

In the formula:

$$
\left\{\begin{array}{l}
\boldsymbol{e}_{k+1}^{(s r)}=\boldsymbol{z}_{k+1}-\boldsymbol{H}(s, k+1) \boldsymbol{x}(k+1 \mid r, k)-\boldsymbol{\varepsilon}(s, k+1) \\
\boldsymbol{\Xi}_{e k+1}^{(s r)}=\boldsymbol{H}(s, k+1) \boldsymbol{P}(k+1 \mid r, k) \boldsymbol{H}^{T}(s, k+1)+\boldsymbol{R}(s, k+1) \\
\boldsymbol{K}_{k+1}^{(s r)}=\boldsymbol{P}(k+1 \mid r, k) \boldsymbol{H}^{T}(s, k+1)\left[\boldsymbol{\Xi}_{e k+1}^{(s r)}\right]^{-1}
\end{array}\right.
$$

Calculation of initial conditions

$$
\begin{gathered}
\boldsymbol{e}_{0}^{(s)}=\boldsymbol{z}(0)-\boldsymbol{H}(s, 0) \boldsymbol{m}_{0}^{(s)}-\boldsymbol{\varepsilon}_{0}^{(s)} \\
\boldsymbol{\Xi}_{e 0}^{(s)}=\boldsymbol{H}(s, 0) \Theta_{0}^{(s)} \boldsymbol{H}^{T}(s, 0)+\boldsymbol{R}(s, 0)
\end{gathered}
$$




$$
\begin{gathered}
\boldsymbol{x}(0 \mid s, 0)=\boldsymbol{m}_{0}^{(s)}+\boldsymbol{K}_{0}^{(s)} \boldsymbol{e}_{0}^{(s)} \\
\boldsymbol{P}(0 \mid s, 0)=\left[\boldsymbol{I}-\boldsymbol{K}_{0}^{(s)} \boldsymbol{H}(s, 0)\right] \boldsymbol{\Theta}_{0}^{(s)} \\
\boldsymbol{K}_{0}^{(s)}=\boldsymbol{\Theta}_{0}^{(s)} \boldsymbol{H}^{T}(s, 0)\left[\boldsymbol{\Xi}_{e 0}^{(s)}\right]^{-1} \\
f[s(0)=i]=\frac{q[s(0)=i] N\left[\boldsymbol{e}_{0}^{(s)} \mid 0, \boldsymbol{\Xi}_{e_{0}^{(s)}}^{(s)}\right.}{\sum_{j=1}^{M} q[s(0)=j] \boldsymbol{N}\left[\boldsymbol{e}_{0}^{(s)} \mid 0, \boldsymbol{\Xi}_{e_{0}^{(s)}}^{(s)}\right.} \quad(i=1,2, \mathrm{~L}, M)
\end{gathered}
$$

When the system structure jumps, the above described model is no longer linear but nonlinear. In order to achieve linearization, centering on $\hat{\boldsymbol{x}}(k+1 \mid r, k)$, expanding the nonlinear model with Taylor series and omitting high-order components ,we can get

$$
\begin{aligned}
\mathbf{z}(\mathrm{k}+1)= & \mathbf{h}(\mathrm{s}, \mathbf{x}(\mathrm{k}+1))+\mathbf{v}(\mathrm{s}, \mathrm{k}+1) \\
= & \mathbf{h}(\mathrm{s}, \hat{\mathbf{x}}(\mathrm{k}+1 \mid \mathrm{r}, \mathrm{k}))+\left.\frac{\partial \mathbf{h}}{\partial \mathbf{x}}\right|_{\mathbf{x}=\hat{\mathbf{x}}(\mathrm{k}+1 \mid \mathrm{r}, \mathrm{k})}(\mathbf{x}(\mathrm{k}+1) \\
& -\hat{\mathbf{x}}(\mathrm{k}+1 \mid \mathrm{r}, \mathrm{k}))+\mathbf{v}(\mathrm{s}, \mathrm{k}+1)
\end{aligned}
$$

The difference between the target measured value and the predicted value in the spherical coordinate system is

$$
\begin{aligned}
& \boldsymbol{z}(k+1)=\boldsymbol{z}(k+1)-\hat{\boldsymbol{z}}(k+1 \mid k) \\
& =\left.\frac{\partial \boldsymbol{h}}{\partial \boldsymbol{x}}\right|_{x=\hat{\boldsymbol{x}}(k+1 \mid r, k)}(\boldsymbol{x}(k+1)-\hat{\boldsymbol{x}}(k+1 \mid r, k))+\boldsymbol{v}(s, k+1)
\end{aligned}
$$

If let

$$
\begin{gathered}
\boldsymbol{H}(s, k+1)=\left.\frac{\partial \boldsymbol{h}}{\partial \boldsymbol{x}}\right|_{\hat{\boldsymbol{x}}(k+1 \mid r, k),}, \\
\boldsymbol{x}(k+1 \mid k)=\boldsymbol{x}(k+1)-\hat{\boldsymbol{x}}(k+1 \mid r, k),
\end{gathered}
$$

we can get

$$
\boldsymbol{z}(k+1)=\boldsymbol{H}(s, k+1) \boldsymbol{x}(k+1 \mid k)+\boldsymbol{v}(s, k+1)
$$

Among them, the specific form of $\boldsymbol{H}(s, k+1)$ is shown in literature[1].

After Tyler expands, Equation (5) changes to

$$
\begin{aligned}
& f\left[z_{k+1} \mid \boldsymbol{x}(k+1), s\right] \\
& =\boldsymbol{N}\left[\boldsymbol{z}_{k+1} \mid \boldsymbol{h}(s, \boldsymbol{x}(k+1 \mid s, k))+\boldsymbol{\varepsilon}(s, k+1), \boldsymbol{R}(s, k+1)\right]
\end{aligned}
$$

Change (17) to:

$$
\left\{\begin{array}{l}
\boldsymbol{e}_{k+1}^{(s r)}=\boldsymbol{z}_{k+1}-\boldsymbol{h}(s, \boldsymbol{x}(k+1 \mid r, k))-\boldsymbol{\varepsilon}(s, k+1) \\
\boldsymbol{\Xi}_{e k+1}^{(s r)}=\boldsymbol{H}(s, k+1) \boldsymbol{P}(k+1 \mid r, k) \boldsymbol{H}^{T}(s, k+1)+\boldsymbol{R}(s, k+1) \\
\boldsymbol{K}_{k+1}^{(s r)}=\boldsymbol{P}(k+1 \mid r, k) \boldsymbol{H}^{T}(s, k+1)\left[\boldsymbol{\Xi}_{e k+1}^{(s r)}\right]^{-1}
\end{array}\right.
$$

The calculation of the initial condition $\boldsymbol{e}_{0}^{(s)}$ changes to

$$
\boldsymbol{e}_{0}^{(s)}=\boldsymbol{z}(0)-\boldsymbol{h}\left(s, \boldsymbol{m}_{0}^{(s)}\right)-\boldsymbol{\varepsilon}_{0}^{(s)}
$$

In the above analysis process, the effective switching between the two models is achieved by a nonlinear Gaussian Approximation filter algorithm. Using the calculated probability weights to synthesize the output of these models, the accuracy and convergence of the filtering estimation are improved.

\section{APPLiCATION EXAMPLES OF ANTI-INTERFERENCE TRACKING ALGORITHM}

\section{A. Building a Model}

In the following, the above filtering algorithm is applied to a system with a pointed interference signal in observation noise, and anti-interference tracking of the maneuvering target is achieved.

Assuming that the target is moving in a plane with constant acceleration, the scan period of both sensors is T. Establishing a uniform acceleration model for a maneuvering target, then we can get the equation of motion as

$$
\boldsymbol{x}(k+1)=\boldsymbol{\Phi} \boldsymbol{x}(k)+\boldsymbol{G} \boldsymbol{w}(k)
$$

$\boldsymbol{w}(k)$ is a Gaussian random sequence with zero mean and variance $\boldsymbol{Q} \cdot w_{1}, w_{2}$ are independent and have the same variance $\sigma_{\alpha}^{2}$.

Therefore,

$$
\boldsymbol{Q}=\sigma_{\alpha}^{2} \boldsymbol{I}
$$

that is $E[\boldsymbol{w}(k)]=\mathbf{0}, E\left[\boldsymbol{w}(k) \boldsymbol{w}^{\mathrm{T}}(j)\right]=\boldsymbol{Q} \delta_{k j}$ The specific form of equation (24) has been discussed in the general study of maneuvering target tracking algorithms.

Due to the mutual switching between the two sensors, tip interference must occur at the instant of switching. The observation equation in this case is: 


$$
\boldsymbol{z}_{1}(k+1)=\boldsymbol{h}_{1}(\boldsymbol{x}(k+1))+\boldsymbol{v}_{1}(s, k+1) \quad(s \in S=1,2)
$$

In the formula,

$$
\boldsymbol{z}_{1}(k+1)=\left[\begin{array}{c}
R(k+1) \\
\theta(k+1)
\end{array}\right], \boldsymbol{h}_{1}(\boldsymbol{x}(k+1))=\left[\begin{array}{c}
\sqrt{x^{2}(k+1)+y^{2}(k+1)} \\
\arctan \frac{y(k+1)}{x(k+1)}
\end{array}\right]
$$

$\boldsymbol{v}_{1}(s, k+1)$ is white noise, $\boldsymbol{v}_{1}(2, k+1)$ is tip interference signal, The measurement of another sensor is not affected by it, and the measurement noise $v_{2}$ is Gaussian white noise with a mean of zero and a variance of $R_{2}(k)$.

The initial conditions for system noise and observed noise and system state are

$$
\begin{aligned}
& \boldsymbol{w}(k) \sim \boldsymbol{N}[\boldsymbol{w}(k) \mid 0, \boldsymbol{Q}] \\
& \boldsymbol{x}_{0}^{(s)} \sim \boldsymbol{N}\left[\boldsymbol{x}_{0}^{(s)} \mid \boldsymbol{m}_{0}^{(s)}, \Theta_{0}^{(s)}\right] \\
& \boldsymbol{v}_{1}(1, k) \sim \boldsymbol{N}\left[\boldsymbol{v}_{1}(1, k) \mid \mathbf{0}, \boldsymbol{R}_{1}(1, k)\right] \\
& \boldsymbol{v}_{1}(2, k) \sim \boldsymbol{N}\left[\boldsymbol{v}_{1}(2, k) \mid \mathbf{0}, \boldsymbol{R}_{1}(2, k)\right] \\
& \boldsymbol{R}_{1}(1, k)<<\boldsymbol{R}_{1}(2, k)
\end{aligned}
$$

The transition probability of the structural state is

$$
q^{(s r)}(k+1, k)=q(s, k+1 \mid r, k)=q(s) \quad(s=1,2)
$$

Assume that the probability of a sharp interference signal in observed noise $q(s=2)=\lambda<<1$

\section{B. Simulation Analysis}

Take $\quad \boldsymbol{R}_{1}(1, k)=\operatorname{diag}\left[0.36 \times 10^{4}(\mathrm{~m})^{2}, 3 \times 10^{-6}(\mathrm{rad})^{2}\right] \quad$, $T=20 \mathrm{~ms}, \lambda=0.05$,

$m_{0}^{s}=\left[\begin{array}{llllll}10000 \mathrm{~m} & 300 \mathrm{~m} / \mathrm{s} \quad 4000 \mathrm{~m} \quad 150 \mathrm{~m} / \mathrm{s} \quad 5 \mathrm{~m} / \mathrm{s}^{2} \quad 4 \mathrm{~m} / \mathrm{s}^{2}\end{array}\right]$ $\sigma_{\alpha}^{2}=100 R_{2}(k)=1 \times 10^{-6}(\mathrm{rad})^{2}$,

$$
\boldsymbol{R}_{1}(2, k)=\operatorname{diag}\left[9.0 \times 10^{4}(\mathrm{~m})^{2}, 7.5 \times 10^{-6}(\mathrm{rad})^{2}\right],
$$

The probability of having and without interference at the initial time is 0.1 and 0.9 respectively. Its transition probability matrix is

$$
q(s, r)=\left\{\begin{array}{l}
{\left[\begin{array}{ll}
0.08 & 0.92 \\
0.05 & 0.95
\end{array}\right], 400<k \leq 600} \\
{\left[\begin{array}{ll}
0.96 & 0.04 \\
0.97 & 0.03
\end{array}\right], k \leq 400, k>600}
\end{array}\right.
$$

Figure I and Figure II compare random interference and non-interference in terms of the RMS estimation of the $x$ direction position and the RMSE of the $x$-direction velocity, respectively.

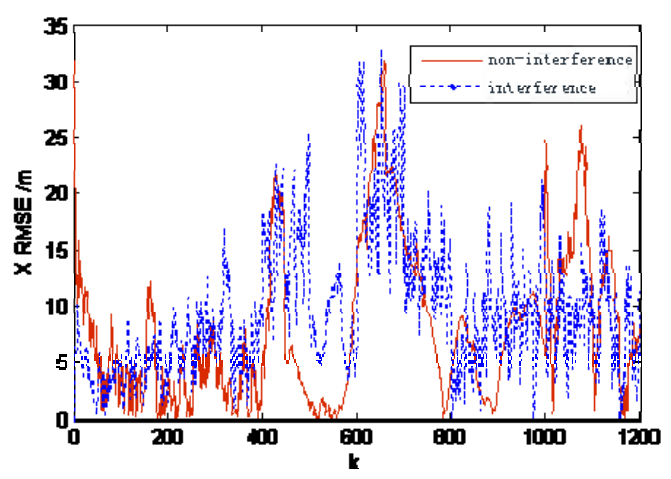

FIGURE I. X POSITION ESTIMATE RMSE

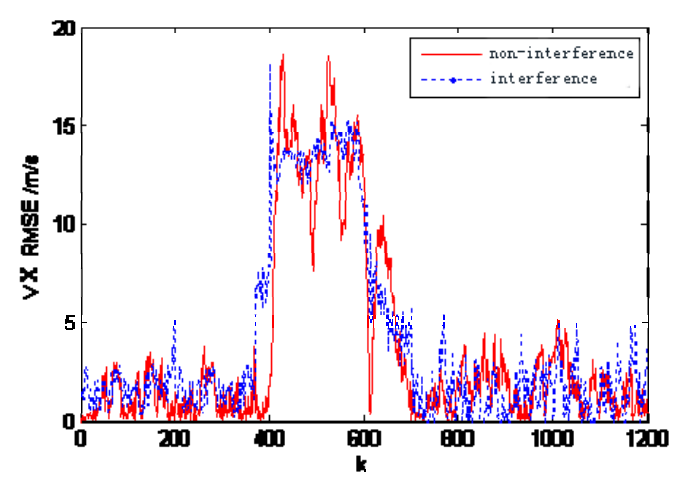

FIGURE II. X DIRECTION SPEED ESTIMATION RMSE

It can be seen from the above that after the interference exists, by using the mutual switching between the two sensors, a level equivalent to the RMSE of position estimation and velocity estimation in the absence of interference is achieved. It shows that the random jump theory can effectively suppress the interference and solve the unstable tracking problem caused by the mutual switching between two different sensors.

\section{REFERENCES}

[1] Blom H A P, Bar-Shalom Y. The interacting multiple model algorithm for system with markovian switching coefficients [J]. IEEE Transactions on Automatic Control, 1988, Vol: 33, No.8: 780-783.

[2] Y. Guo and B. Huang, Moving horizon estimation for switchin nonlinear systems [J]. Automatic , 2013,49(11):3270-3281.

[3] Gordon N J ,Salmond D J,Smith A F M.Novel approach to nonlinear/non Guassian Bayesian state estimation [J].IEEE Procee Dings F,1993,140(2):107 113.

[4] Wu Wen Rong,Cheng Peen Pau. A nonlinear IMM algorithm for maneauvering target tracking [J].IEE TAES,1994,30(3):25 36.

[5] COSTA O L V,FRAGOSO M D,MARQUES R P.Discrete Time Markov Jump Linear Systems [M].London:Springer-Verlag,2005.

[6] ORGUNER U,DEMIREKLER M.Risk-sensitive filtering for jump Markov linear systems [J].Automatica,2008,44(1):109-118.

[7] BUKHALEV V A.An optimal control for stochasic jump system [J].Theory of Controls,1992,(4):88-97(in Russian). 